\title{
Fermi-surface analysis of a quasi-two-dimensional monophosphate tungsten bronze
}

\author{
L. Roca, ${ }^{1,2}$ A. Mascaraque,,${ }^{1, *}$ J. Avila,,${ }^{1,2}$ S. Drouard,${ }^{3}$ H. Guyot,${ }^{3}$ and M. C. Asensio ${ }^{1,2}$ \\ ${ }^{1}$ LURE, Centre Universitaire Paris-Sud, Bâtiment 209D, B.P. 34, 91898 Orsay Cedex, France \\ ${ }^{2}$ Instituto de Ciencia de Materiales, CSIC, 28049 Madrid, Spain \\ ${ }^{3}$ LEPES-CNRS, B.P. 166, 38042 Grenoble, Cedex 9, France
}

(Received 16 June 2003; revised manuscript received 15 October 2003; published 27 February 2004)

\begin{abstract}
The $\mathrm{P}_{4} \mathrm{~W}_{8} \mathrm{O}_{32}$ compound is a member of the low-dimensional monophosphate tungsten bronzes family whose reduced dimensionality induces electronic instabilities such as charge-density waves (CDWs). We report here the direct mapping of the Fermi surface (FS) of this compound at room temperature using synchrotronradiation angle-resolved photoemission. The recorded FS images confirm the superimposition of three nested sheets, as proposed by the hidden-nesting model. We found two well defined parallel stripes along the $\overline{\Gamma Y}$ direction as well as a crosslike feature. Moreover, the small FS splitting predicted by $a b$ initio calculations was distinguished in the experimental data. To extract quantitative information on the CDW phase transitions, the values of the nesting vectors were also determined from the FS topology. The obtained values were in excellent agreement with existing tight-binding calculations, although the accord was even better with the recently published ab initio theoretical predictions.
\end{abstract}

DOI: 10.1103/PhysRevB.69.075114

PACS number(s): 79.60.-i, 71.45.Lr, 64.60.-i, 71.18.+y

\section{INTRODUCTION}

Low-dimensional systems have attracted much attention in recent years because of their unusual chemical and physical properties, which initiated a lot of theoretical works. ${ }^{1,2}$ In these systems, a strong structural anisotropy is reflected in the electronic structure that exhibits uncommon features such as low-dimensional transport, anomalous magnetic properties, metal-insulator and metal-metal transitions, and periodic lattice distortions. ${ }^{3}$

In general, low-dimensional metallic oxides exhibit two types of electronic instabilities: charge-density wave (CDW) or superconductivity. The presence of these electronic instabilities is closely related to the anisotropy of the Fermi surface (FS). Regarding the CDW, it is characterized by a structural $\vec{q}=2 \vec{k}_{F}$ modulation. This structural modulation should be associated with a FS that presents enough nesting in the normal state, i.e., large flat areas connected by a $\vec{q}=2 \vec{k}_{F}$ vector. This specific shape of the FS allows a coupling between the vibrational modes of the lattice and the electrons, leading to the formation of electron-hole pairs at the Fermi energy, and corresponds to the tendency of materials to decrease their electronic energy by opening a gap. The coupling between the lattice and the electron gas leads to the CDW state which is a periodic lattice distortion coupled to an electronic density modulation.

The main families of CDW materials are charge-transfer organic salts, ${ }^{4,5}$ transition-metal chalcogenides, ${ }^{6,7}$ and molybdenum and tungsten bronzes. ${ }^{2}$ Several works have focused on the study of monophosphate tungsten bronzes (MPTB), which is a family of low-dimensional conductors with the general formula $\left(\mathrm{PO}_{2}\right)_{4}\left(\mathrm{WO}_{3}\right)_{p}\left(\mathrm{WO}_{3}\right)_{q}$. The essential building units of these materials are $\mathrm{WO}_{3}$ octahedra sharing $\mathrm{O}$ corners to form $\mathrm{W}-\mathrm{O}$ step layers. Two neighboring layers are linked across $\mathrm{PO}_{4}$ tetrahedra planes (Fig. 1). ${ }^{2}$

Electron and $\mathrm{x}$-ray diffraction studies have shown that several members of the MPTB family exhibit low- (a)

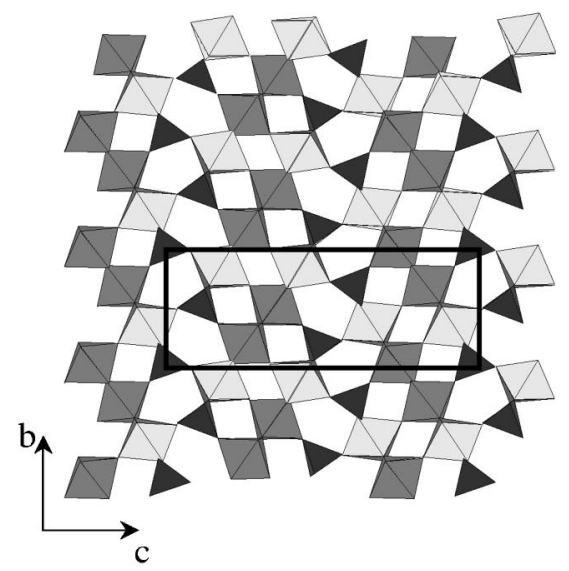

(b)

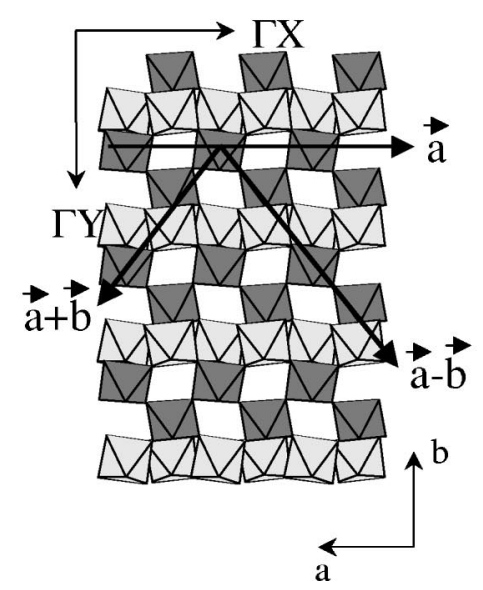

FIG. 1. Projections of the structure of the $\left(\mathrm{PO}_{2}\right)_{4}\left(\mathrm{WO}_{3}\right)_{4}\left(\mathrm{WO}_{3}\right)_{4}$ compound along two of the crystallographic directions. (a) Projection along $\vec{a}$ shows perovskite-type layers made up of $\mathrm{WO}_{6}$ octahedra and linked across $\mathrm{PO}_{4}$ tetrahedra, represented here as triangles. Pentagonal tunnels are formed in the junction between two W-O layers. (b) Projection along $\vec{c}$ shows $1 \mathrm{D}$ chains formed by $\mathrm{WO}_{6}$ octahedra along $\vec{a}$ and $\vec{a} \pm \vec{b}$ directions. 
temperature periodic lattice distortions. In the particular case of the MPTB family members where $p=q=m \leqslant 6$, the CDW phase transition is theoretically explained by the hidden-nesting mechanism. ${ }^{8}$ This model ${ }^{9-12}$ describes the FS as a superimposition of different sheets related to the onedimensional (1D) chains that form the MPTB structure. The CDW instabilities detected in these materials would be the result of the different nesting wave vectors associated with these 1D chains. The hidden-nesting mechanism has been directly observed by Gweon and co-workers in the FS of the low-dimensional molybdenum oxides $\mathrm{NaMo}_{6} \mathrm{O}_{17}$ and $\mathrm{KMo}_{6} \mathrm{O}_{17} \cdot{ }^{13}$

The full understanding of the electronic properties of the MPTB compounds requires the complete comprehension of their FS. This is crucial because the CDW wave vector $\vec{q}$ is determined by the FS topology. The most suitable technique to obtain the FS map of these compounds is angle-resolved photoemission spectroscopy (ARPES). Two complementary methods can be used. The first one consists in following the dispersion of the bands from energy distribution curves, and obtaining from them the $k$-space point where a band crosses the Fermi level. The second method uses a 2D map of the FS obtained by monitoring the photoemission intensity in a narrow window centered at the Fermi level, as a function of emission angles. This second method has proved recently to be extremely effective in detecting even tiny features at the FS. In our analysis, we have used this automatic scanning method, where the intensity at the Fermi level is recorded along a series of azimuthal scans, each step being repeated at different polar angles within a given photon energy.

In this paper, we have studied the $m=4$ member of the $\left(\mathrm{PO}_{2}\right)_{4}\left(\mathrm{WO}_{3}\right)_{2 m}$ family, which exhibits two Peierls transitions at $\sim 80 \mathrm{~K}$ and $\sim 52 \mathrm{~K} .{ }^{14}$ Both phase transitions are of second order. X-ray diffraction data have shown that they correspond to an incommensurate CDW (Refs. 15-17) with two vectors: $\vec{q}_{1}=[0.330(5), 0.295(5), 0]$ and $\vec{q}_{2}$ $=[0.340(5), 0,0]$ in reciprocal lattice units. Each transition coincides with an anomaly in the resistivity curve and with a decrease of the magnetic susceptibility, both related to the opening of partial gaps at the Fermi level. In spite of the large number of works devoted to the MPTB compounds, their electronic band structure has only recently been investigated experimentally. ${ }^{18}$ In order to obtain a deeper understanding of the electronic properties, we have measured the FS at room temperature throughout a large $k$ space and we have determined the nesting wave vectors responsible for the two CDW phase transitions. The results allow to establish a direct link between the resistivity anomalies and the electronic behavior of the compound. The obtained data agree quite well with the existing theoretical calculations, which support that the CDW transition is based on a hidden-nesting mechanism.

\section{EXPERIMENT}

The experiments were done in the SU8 beamline at LURE (Orsay) receiving synchrotron radiation from Super Aco storage ring. The measurements were taken at room temperature, consequently above the critical temperature of the studied samples. The typical size of the samples was $2 \times 2 \mathrm{~mm}^{2}$, and they were grown using the chemical vapor transport technique. ${ }^{19}$ The crystals were cleaved in situ in ultra high vacuum (base pressure $5 \times 10^{-11} \mathrm{mbar}$ ). The chamber was equipped with a 50-mm hemispherical VSW angle-resolving analyzer mounted on a two-axes manipulator with an overall angular resolution of $0.5^{\circ}$. The FS was measured with $45^{\circ}$ polar angle range and $360^{\circ}$ azimuthal angle range. For the selected energy $h \nu=30 \mathrm{eV}$, typical energy resolution was of $50 \mathrm{meV}$. The quality of the sample was monitored during the data acquisition by measuring the spectral intensity between the conduction and the valence band. The orientation process was done in two steps using $\mathrm{x}$-ray based techniques in order to avoid any electron damage of the samples by electron bombardment. ${ }^{20}$ All the samples were aligned using x-ray photoelectron diffraction, which provides a very precise method to determine the high-symmetry directions, and the normal to the surface. Lately, after all the samples were studied, their orientation was confirmed by low-energy electron diffraction.

\section{CRYSTALLOGRAPHIC STRUCTURE}

MPTB have been synthesized and their structures have been studied for more than 20 years. The small $m$ members crystallize in an orthorhombic lattice. The structure is built up from perovskite-like $\mathrm{ReO}_{3}$-type infinite layers of $\mathrm{WO}_{3}$ octahedra stacked along the orthorhombic (001) direction [Fig. 1(a)]. The $\mathrm{WO}_{3}$ octahedra are linked together forming chains running along $\vec{a}$ and $\vec{a} \pm \vec{b}$ crystallographic directions [Fig. 1(b)]. Neighboring step layers are connected by planes of $\mathrm{PO}_{4}$ tetrahedra, giving rise to pentagonal-shape tunnels in the junctions (Fig. 1).

The $p$ and $q$ indices of the $\left(\mathrm{PO}_{2}\right)_{4}\left(\mathrm{WO}_{3}\right)_{p}\left(\mathrm{WO}_{3}\right)_{q}$ general formula indicate the number of $\mathrm{WO}_{3}$ octahedra per unit cell needed to form the W-O layers. In the particular case $p=q$ $=m$, the general formula is reduced to $\left(\mathrm{PO}_{2}\right)_{4}\left(\mathrm{WO}_{3}\right)_{2 m}$. In the $\left(\mathrm{PO}_{2}\right)_{4}\left(\mathrm{WO}_{3}\right)_{8}$ compound, each chain is composed of clusters of $m=4$ corner-sharing octahedra. The $\mathrm{W}_{4} \mathrm{O}_{16}$ step layers of the $m=4$ member are the result of chain condensation, where the first octahedron of one chain shares the axial oxygen atom with the third octahedron of the following chain (13). During the formation of the $\mathrm{W}_{4} \mathrm{O}_{18}$ chain, the tungsten octahedra suffer a distortion to reduce the strain. ${ }^{17,21}$ This distortion is mainly a rotation around the vertical axis.

Two successive step layers run following a herringbone pattern along the $c$ direction $^{21}$ and they are related by a $2{ }_{1}$-fold axis. The unit-cell sizes are $a=5.285 \AA, b$ $=6.569 \AA$, and $c=17.351 \AA$. Due to the layered character of MPTB compounds, the surface projection of the Brillouin zone (surface Brillouin zone, SBZ) is straightforward: the rectangle formed by $\Gamma, X, Y$, and $S$ bulk symmetry points becomes the surface Brillouin zone formed by $\bar{\Gamma}, \bar{X}, \bar{Y}$, and $\bar{M}$ symmetry points, respectively. ${ }^{18}$

\section{RESULTS AND DISCUSSION}

The electronic properties of the $\mathrm{WO}_{3}$ layers were first explained by Goodenough ${ }^{22} 40$ years ago. In the 1980s, 


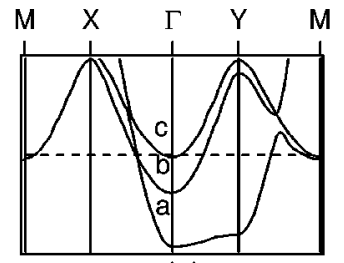

(a)

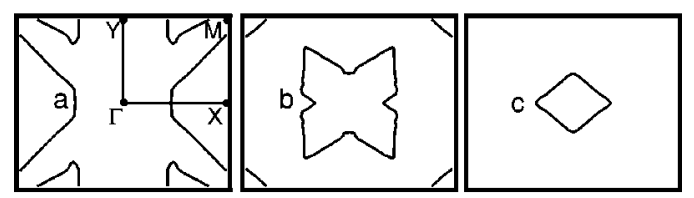

(c)

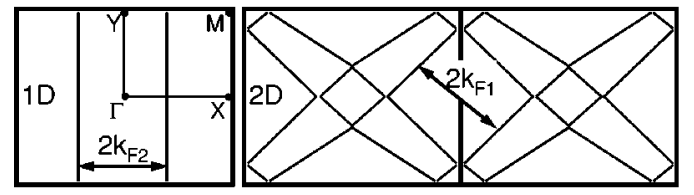

(g) (b)

(h)

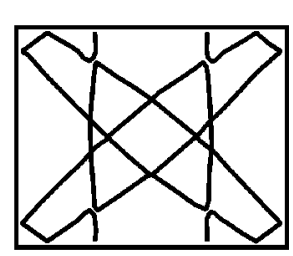

FIG. 2. (a) Dispersion relation of the bottom $t_{2} g$ block bands calculated with tight-binding method in Ref. 12. (b) Theoretical FS obtained from tight-binding calculations (Ref. 10). The FS results from the composition of three different sheets related to each one of the three bands shown in panel (a): band $a$ (c), band $b$ (d), and band $c$ (e). But the FS can also be decomposed in three low-dimensional FS related to the (g) $\vec{a}$ chains, (h) $\vec{a} \pm \vec{b}$ chains that form the compound. The associated nesting vectors, $2 \vec{k}_{F 2}$ and $2 \vec{k}_{F 1}$, have also been represented.

Whangbo and co-workers performed theoretical tightbinding band calculations based upon the extended Hückel method. ${ }^{9-12}$ From these studies they showed that the essential features of the MPTB electronic structure can be explained from ideal $\mathrm{W}_{4} \mathrm{O}_{16}$ step layer calculations. Because this step layer is common to all the compounds of the MPTB family, the electronic structure is very similar in all these materials.

The electronic structure near the Fermi level of this ideal step layer presents two bands, labeled $a$ and $b$, as a consequence of the partial filling of the W $5 d t_{2 g}$-block bands with electrons coming from the $\mathrm{PO}_{2}$ groups. ${ }^{9-\mathrm{f}^{2}}$ When the calculations are performed taking into account the real distortion suffered by the $\mathrm{WO}_{3}$ octahedra [see Fig. 2(a)], the double degeneration of the upper $b$ band is lifted in bands $b$ and $c$. The dispersive nature of these bands is due to the interaction between the $\mathrm{W}_{4} \mathrm{O}_{18}$ chains and indicates the two-dimensional character of these bands. On the contrary, the deeper band $a$ has one-dimensional nature, reflected in its nondispersive character along one particular direction. This electronic structure has recently been experimentally confirmed. ${ }^{18}$

As these three bands are partially filled, they are cut by the Fermi level and therefore they contribute to the FS. The different Fermi-surface sheets associated with bands $a, b$, and $c$ are represented in Fig. 2 in panels (c), (d), and (e), respectively. The complete FS of these compounds arises from the combination of these three contributions.

As explained above, it has been proposed that the stabilization of a CDW phase in these compounds is due to a hidden-nesting mechanism. In this model, the FS can be assembled as the addition of different sheets generated by the $1 \mathrm{D}$ chains that form the MPTB compound. Figures $2(\mathrm{~g})$ and 2(h) show the mentioned sheets associated with the $\vec{a}$ chains and $\vec{a} \pm \vec{b}$ chains, respectively. Because there is no symmetry relationship between the two $1 \mathrm{D}$ chain directions, there are two critical wave vectors related to each FS sheet, represented in the figure as $2 k_{F 2}$ and $2 k_{F 1}$, and therefore two different CDW phase transitions. Due to the relatively complicated shape of the FS, the nesting is not perfect, so only localized gaps may open and therefore the low-temperature phase remains metallic.

More recently $a b$ initio density-functional theoretical calculations have been performed for the $m=4$ member. $^{23}$ The electronic structure obtained is similar, except for the interlayer splitting, to that calculated with the extended Hückel method and corroborates the results obtained by Whangbo and co-workers, giving rise to a FS that confirms the hiddennesting model.

\section{A. The Fermi surface}

The experimental FS contour is shown in Fig. 3. The image does not include any symmetrizations, therefore each point of the $360^{\circ}$ image corresponds to measured data. The intensities were normalized with respect to the photon flux and represent a 2D image of the photoemission intensity at the Fermi level. ${ }^{24}$ The image is plotted in a parallel momentum $\left(k_{\|}\right)$projection to facilitate the comparison with the theory. Normal emission corresponds to the center of the image. The data were taken with $30 \mathrm{eV}$ photon energy in even detection geometry. ${ }^{18}$ Using tunable synchrotron radiation it is possible to obtain a bulk FS slice by slice. However, since in this compound the $5 d$ conduction electrons are located in the perovskite $\left(\mathrm{WO}_{3}\right)$-type slabs, the electronic properties are quasicylindrical and the FS can be described as a weakly corrugated cylinder. Thus, besides effects due to the energy-dependent cross section of the initial states and to final state effects, one photon energy should be enough to obtain the whole relevant information.

As can be seen in Fig. 3, nine different SBZ's were probed in the experiment, which have been labeled by their corresponding $\bar{\Gamma}$ points. Bands $a, b$, and $c$ [see Fig. 2(a)] cross the Fermi level, contributing to the FS. The bandwidths of these three bands are relatively large. ${ }^{18,25}$ This fact is reflected in the shape of the FS features, which are much broader than expected from theoretical calculations.

Some remarkable features are observed in the 2D FS mapping of Fig. 3. The most relevant ones are two parallel bands that run along the $\overline{\Gamma Y}$ direction. They are clearly detected in all SBZ's, but they exhibit maximum intensity in $\bar{\Gamma}_{01}$ and $\bar{\Gamma}_{0 \overline{1}}$ SBZ's, where they become the most prominent features of the image.

Additional diagonal bright stripes running from $\bar{M}$ points to $\bar{\Gamma}$ points are also detected. These stripes form a crosslike shape. Although they are observed in all SBZs, they are specially intense in the inner corners of $\bar{\Gamma}_{\overline{1} 1}, \bar{\Gamma}_{11}, \bar{\Gamma}_{\overline{1} \overline{1}}$, and $\bar{\Gamma}_{1 \overline{1}}$ SBZ's. 


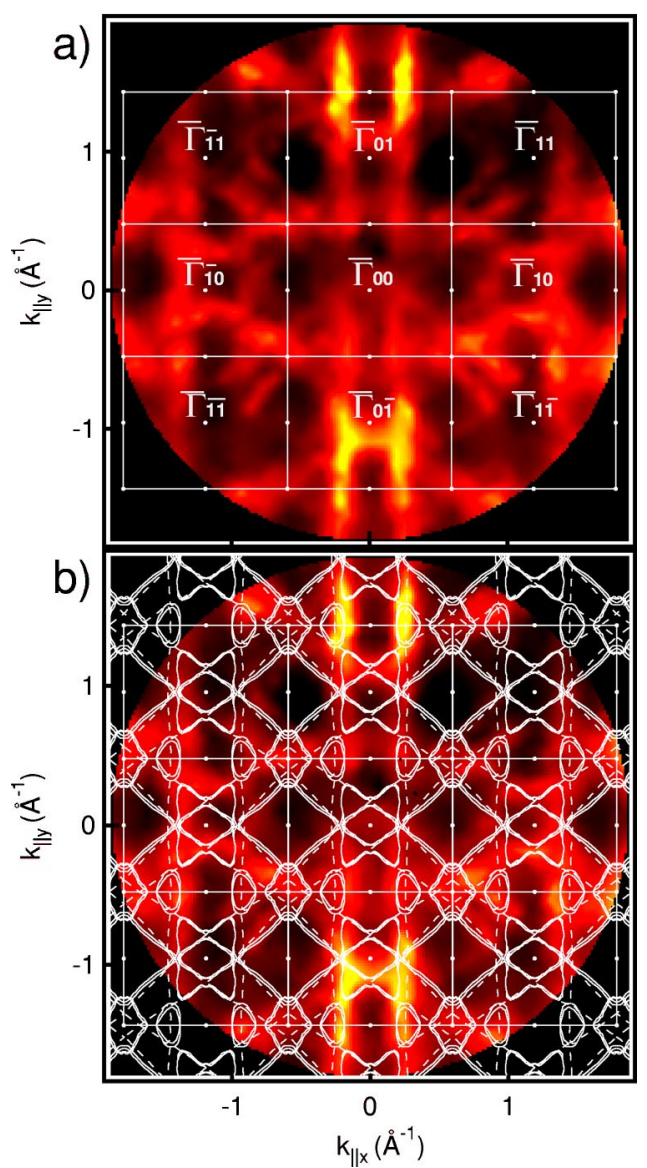

FIG. 3. (Color online) (a) Experimental FS measured in even detection geometry and with $h \nu=30 \mathrm{eV}$. Brighter points correspond to maxima of the photoemission intensity, while the darker ones correspond to minima. The values of $\bar{\Gamma}$ points indicate different SBZ's zones. (b) The same experimental data compared with existing theoretical FS: dashed lines reproduce the FS predicted in Ref. 10 using tight-binding calculations; solid lines correspond to the theoretical results obtained from $a b$ initio calculations in Ref. 23.

An interesting property of this FS image is related to the absolute intensity of the two different features. When the parallel bars are more intense in a particular SBZ, the crosslike feature is more difficult to see and vice versa. In $\bar{\Gamma}_{\overline{1} 1}$, $\bar{\Gamma}_{11}, \bar{\Gamma}_{\overline{1} \overline{1}}$, and $\bar{\Gamma}_{1 \overline{1}}$ SBZ's, the parallel bands look weaker, but the crosslike feature is easier to see. On the contrary, in $\bar{\Gamma}_{01}$ and $\bar{\Gamma}_{01}$ SBZ's, the parallel bands are more intense, hiding the crosslike feature. Finally, both structures are also observed in $\bar{\Gamma}_{10}$ and $\bar{\Gamma}_{\overline{1} 0}$ SBZ's, but much weakly than in previous SBZ's.

All these variations between the intensity ratios of the features in different SBZ's are just a consequence of BZ selection rules, common in many systems. ${ }^{26}$ The spectral intensity obtained in photoemission is not simply proportional to the spectral function, but it is weighted by the electronphoton matrix element. This matrix element is strongly $\vec{k}$ dependent, so it will affect different SBZ's unequally, provoking the change in their relative spectral intensity. ${ }^{27,28}$

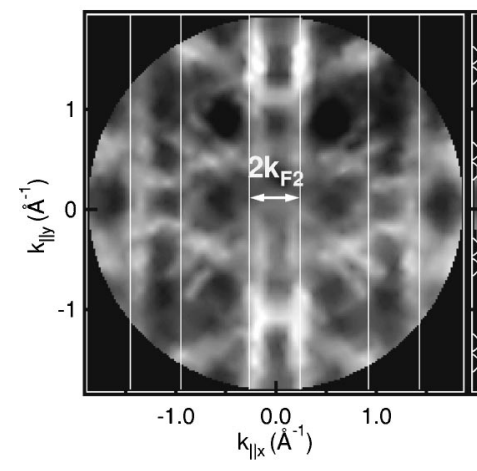

(a)

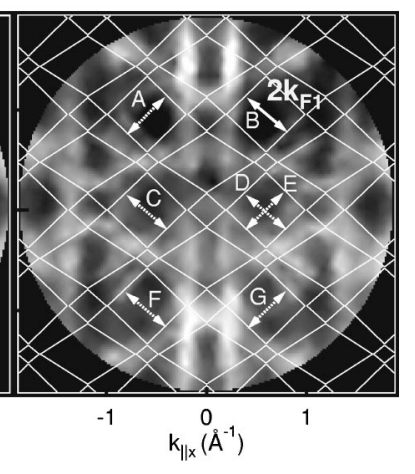

(b)
FIG. 4. Experimental and theoretical FS sheets resulting from hidden-nesting model and related to (a) $\vec{a}$ chains and (b) $\vec{a} \pm \vec{b}$ chains. In both cases, the corresponding nesting vectors, $2 \vec{k}_{F 2}$ and $2 \vec{k}_{F 1}$, have been represented. In panel (b), the different arrows labeled with letters from $A$ to $G$ point out the different cuts that have been made in order to extract the value of $2 \vec{k}_{F 1}$.

The mentioned different intensity ratio of two FS sheets in different SBZ's supports the hidden-nesting model too. This theoretical model is correct only under the hypothesis of a weak interaction between different chains, which brings a weak orbital hybridization between relevant chain orbitals. This assumption may be reasonable for this compound, since the three chains are related to different W $5 d$ orbitals. If the two FS sheets come from chains not related by any symmetry elements, it is reasonable to expect a different behavior of the matrix element, and so different intensity dependence in different SBZ's.

Figure 3(b) shows the 2D FS image together with the theoretical calculations from Refs. 10 and 23. Both calculated FS's are similar, but as will be discussed later, they exhibit some important differences. The consideration of the two theoretical calculations is very useful for our discussion, because although the $a b$ initio predictions are more detailed and exhibit more structure, the tight-binding results are helpful to understand the orbital origin of the different features.

In Fig. 4, the experimental results are plotted together with the decomposition of the FS in the different sheets as is proposed in the hidden-nesting model [see Figs. 2(g,h)]. The parallel bands running along the $\overline{\Gamma Y}$ direction coincide very well with the 1D FS sheet related to $\vec{a}$ chains [Fig. 4(a)]. In spite of their lateral modulation, their shape is very suitable for nesting. The $2 \mathrm{D}$ crosslike features correspond to the chains running along $\vec{a} \pm \vec{b}$ directions [Fig. 4(b)]. They also present parallel areas that can contribute to nesting.

Figure 5 displays an enlarged representation of the $\bar{\Gamma}_{01}$ SBZ. Panel (a) shows the theoretical calculations together with the measured FS in this region. It can be observed that this SBZ is dominated by the parallel lines coming from $\vec{a}$ chains. These sheets are observed as wide stripes centered around the vertical theoretical lines. As mentioned above, the experimental width of the stripes is due to the large linewidth of the electronic states. ${ }^{18}$ 


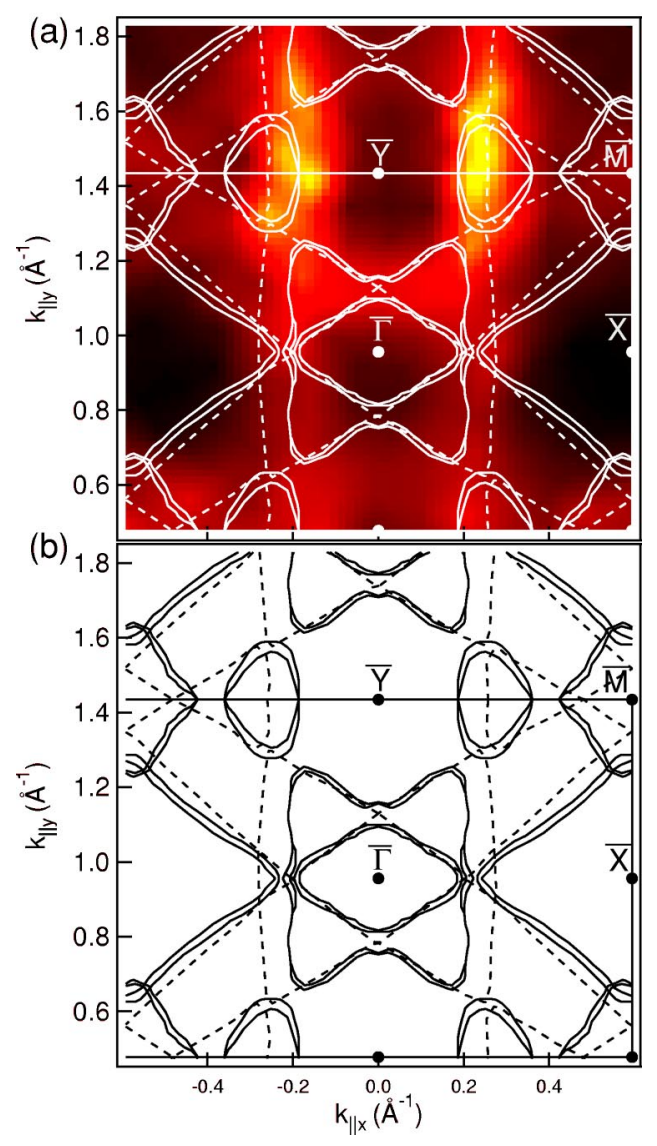

FIG. 5. (Color online) Detailed representation of $\bar{\Gamma}_{01}$ SBZ. Panel (a) shows the theoretical calculations from Refs. 10 and 23 as dashed and solid lines, respectively. Panel (b) shows the same theoretical predictions along with the experimental results measured in the corresponding region.

In this detailed image a lateral modulation in the parallel lines is observed. The brightest areas correspond to their intersection with the crosslike feature. The cross is also detected as an increase of the intensity around $\bar{M}$ points. An intensity depletion is also observed around $\bar{X}$ and $\bar{Y}$ symmetry points.

The agreement between the experimental and the theoretical FS shape is in general very good. However, a squarelike feature is observed centered around the $\bar{\Gamma}$ point, where a rhombus form is expected from theory. The square shape is the result of the large linewidth of the electronic states. ${ }^{18}$

Band $b$ has a parabolic dispersion around $\bar{\Gamma}$ point, very close to the Fermi level [see Fig. 2(a)]. Its large linewidth produces an homogeneous intensity around $\bar{\Gamma}$ when it crosses the Fermi level.

In panel (b), only the theoretical calculations are represented, in order to make a deeper comparison between both of them. Tight-binding predictions from Ref. 10 and $a b$ initio calculations from Ref. 23 are displayed as dashed and solid lines, respectively. From the direct observation we notice that the associated nesting vectors are larger in the former than in the latter case. This is more evident in the case of $2 \vec{k}_{F 2}$, whose values are approximately $0.52 \AA$ and $0.40 \AA$, respectively.

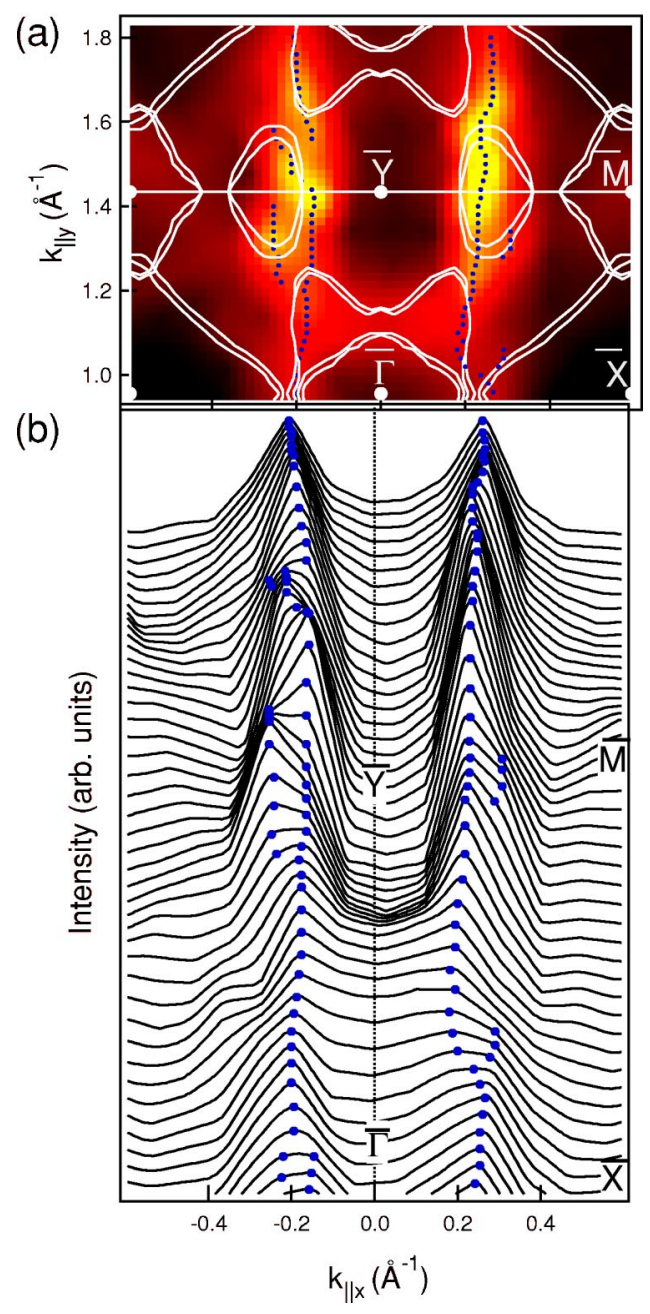

FIG. 6. (Color online) (a) FS map for the region between $\bar{\Gamma}_{01}$ and $\bar{\Gamma}_{02}$ SBZ. Solid white lines correspond to the theoretical calculations from Ref. 23. Points represent the intensity maxima extracted from cuts perpendicular to the $\overline{\Gamma Y}$ direction reproduced in panel (b).

But the most striking difference between both theoretical results is the splitting observed in the ab initio calculations, which is not present in the tight-binding predictions. However, the explanation is very simple: as reported by Sandre et al., this splitting arises from the interlayer coupling in the MPTB compound, so it is not expected to be found in the tight-binding calculations, taking into account that they were performed for a single layer.

This splitting is present in our experimental results. In order to evidence it, different FS cuts were performed along the direction perpendicular to $\overline{\Gamma Y}$, i.e., the $2 \vec{k}_{F 2}$ direction. Some of them, those contained in the zone included between the $\bar{\Gamma}_{01}$ and $\bar{\Gamma}_{02}$ SBZ's, have been reproduced in Fig. 6(b). The intensity maxima in each cut are emphasized with points. We can easily distinguish the splitting, for instance, in the zone close to the $\bar{Y}$ point, in the features located at both sides. Similar behaviors have also been observed in other SBZ's. 
In order to compare the measured splitting with that obtained from the $a b$ initio calculations, Fig. 6(a) shows both results superimposed in the region were cuts in panel (b) were done. The points indicating the intensity maxima for each cut in panel (b) have also been superimposed to this FS map as an eye guide. By direct observation, we can conclude that the splitting predicted by Sandre et al., $\left(\sim 0.02 \AA^{-1}\right)$ is clearly smaller than the experimental one $\left(\sim 0.07 \AA^{-1}\right)$. This can be easily checked in the region situated on the left of the $\bar{Y}$ point. Here the distance between the two sets of points that indicate maxima positions is larger than that between the white lines that represent the ab initio calculations. This means that the interlayer interactions, proposed as the cause of the FS splitting, have been underestimated in the theoretical calculations by Sandre et al.

In addition to this splitting, a detailed study of the FS reveals the presence of a lateral modulation in the straight features that run parallel to the $\overline{\Gamma Y}$ direction. This warping results from the intralayer interaction between the different types of chains, $\vec{a}$ and $\vec{a} \pm \vec{b}$. Its magnitude has been experimentally determined by following the maxima relative to this feature in different FS cuts perpendicular to the $\overline{\Gamma Y}$ direction throughout the $\bar{\Gamma}_{01}, \bar{\Gamma}_{00}$ and $\bar{\Gamma}_{01}$ SBZ's. Some of these cuts are displayed in Fig. 6(b). The modulation obtained is 0.16 $\pm 0.07 \AA^{-1}$. This modulation in the vertical feature coming from the $\vec{a}$ chains is partially affected by the FS sheets coming from $\vec{a} \pm \vec{b}$ chains, as both contributions appear mixed in the regions where they intersect. This involves an interaction between partially filled bands with different orbital origin, whose effect is also shown in the hybridization gaps present in the FS in the places where sheets coming from different bands were supposed to cross. These gaps are a consequence of the fact that two bands avoid to cross themselves in the vicinity of the Fermi level, leading to the opening of these hybridization gaps in the FS. This is in fact the origin of the hidden nesting. ${ }^{8}$

From the comparison between the experimental and the theoretical FS shape, we conclude that 2D FS images exhibit a very good agreement with theoretical calculations, and are in harmony with the hidden-nesting mechanism. However, the existence of the mentioned modulation and the large splitting means that the interlayer interaction in the MPTB compounds is stronger than expected. Therefore this interaction should be taken into account fully in future theoretical calculations, in order to improve the predictions derived from them.

\section{B. The nesting vector}

The nesting of the FS couples unoccupied states at the Fermi level with occupied states at the same energy by a $\vec{q}_{C D W}=2 \vec{k}_{F}$ nesting vector. This produces a divergence in the $\vec{q}$-dependent electron-hole polarizability, leading to a CDW instability. ${ }^{29}$ Therefore, not only the FS shape but also the value of the Fermi wave vector $\left(\vec{k}_{F}\right)$ is fundamental in the understanding of the $\mathrm{CDW}$ transition.

The $\left(\mathrm{PO}_{2}\right)_{4}\left(\mathrm{WO}_{3}\right)_{4}\left(\mathrm{WO}_{3}\right)_{4}$ compound undergoes two consecutive phase transitions at $80 \mathrm{~K}$ and $52 \mathrm{~K}$. If a
TABLE I. Experimental distances obtained from FS map cuts $\left(\left|k_{F 2}\right|\right)$ between the maxima along the $\vec{k}_{F 1}$ direction. $k_{\| y}$ represents the vertical reciprocal point where the cut was done. The values are expressed in $\AA^{-1}$. The experimental error is $\pm 0.07 \AA^{-1}$.

\begin{tabular}{lccc}
\hline \hline$k_{\| y}$ & $2\left|k_{F 2}\right|$ & $k_{\| y}$ & $2\left|k_{F 2}\right|$ \\
\hline 0.60 & 0.36 & -0.60 & 0.34 \\
0.65 & 0.39 & -0.65 & 0.39 \\
0.70 & 0.39 & -0.70 & 0.40 \\
0.75 & 0.37 & -0.75 & 0.43 \\
0.80 & 0.39 & -0.80 & 0.43 \\
0.85 & 0.40 & -0.85 & 0.46 \\
0.90 & 0.45 & -0.90 & 0.42 \\
$0.956(\bar{\Gamma})$ & 0.41 & $-0.956(\bar{\Gamma})$ & 0.42 \\
1.00 & 0.42 & -1.00 & 0.41 \\
1.05 & 0.36 & -1.05 & 0.36 \\
1.10 & 0.31 & -1.10 & 0.35 \\
1.15 & 0.38 & -1.15 & 0.41 \\
1.18 & 0.37 & -1.18 & 0.43 \\
1.20 & 0.39 & -1.20 & 0.42 \\
1.25 & 0.42 & -1.25 & 0.42 \\
1.30 & 0.47 & -1.30 & 0.41 \\
1.35 & 0.44 & -1.35 & 0.40 \\
1.40 & 0.39 & -1.40 & 0.43 \\
1.45 & 0.41 & -1.45 & 0.42 \\
\hline \hline
\end{tabular}

CDW mechanism is behind these transitions, they should have associated $\vec{q}_{1}=2 \vec{k}_{F 1}=(0.330(5), 0.295(5), 0)$ and $\vec{q}_{2}$ $=2 \vec{k}_{F 2}=(0.340(5), 0.000(5), 0)$ nesting vectors, respectively, ${ }^{17}$ where the vectors are expressed in reciprocal lattice units. Alternatively, taking into account the reciprocal lattice vector values $a^{*}=1.189 \AA^{-1}$ and $b^{*}=0.956 \AA^{-1}$, they can also be expressed as $2 \vec{k}_{F 1}=(0.392,0.282,0) \AA^{-1}$, $\left(2\left|\vec{k}_{F 1}\right|=0.482 \AA^{-1}\right)$, and $2 \vec{k}_{F 2}=(0.404,0,0) \AA^{-1}$.

It is possible to extract the Fermi vector values from the experimental data, by linking the most intense lines in the FS topology, which represent the Fermi-level crossing when they are taken in the appropriate direction.

The nesting vector of the second transition corresponds to a charge modulation along the $\vec{a}$ direction, i.e., the parallel lines along $\overline{\Gamma Y}$. In fact, this pair of stripes comes from the 1D chains running along the $\vec{a}$ direction. Then, the Fermi vector can be extracted from the distance between the two maxima obtained in the FS cuts perpendicular to $\overline{\Gamma Y}$. Due to the modulation of this FS sheet, the $k_{\| y}$ component of the total wave vector is affected by the modulation indicated in Fig. 6. In order to get an average value, 150 different FS cuts have been done perpendicular to the $\overline{\Gamma Y}$ direction throughout several BZs. In particular, they have been made in $\bar{\Gamma}_{01}, \bar{\Gamma}_{00}$, and $\bar{\Gamma}_{0 \overline{1}}$ SBZ's, where the parallel features are more intense. The results are shown in Table I and some relevant cuts are represented in Fig. 7(b). From these data we obtain a mean value of $2\left|\vec{k}_{F 2}\right|=0.40 \pm 0.07 \AA^{-1}$. It is in very good agreement with the value obtained with $\mathrm{x}$-ray diffraction, 0.404 $\pm 0.005 \AA^{-1}$ by Ludecke et al. 
(a)

(b)

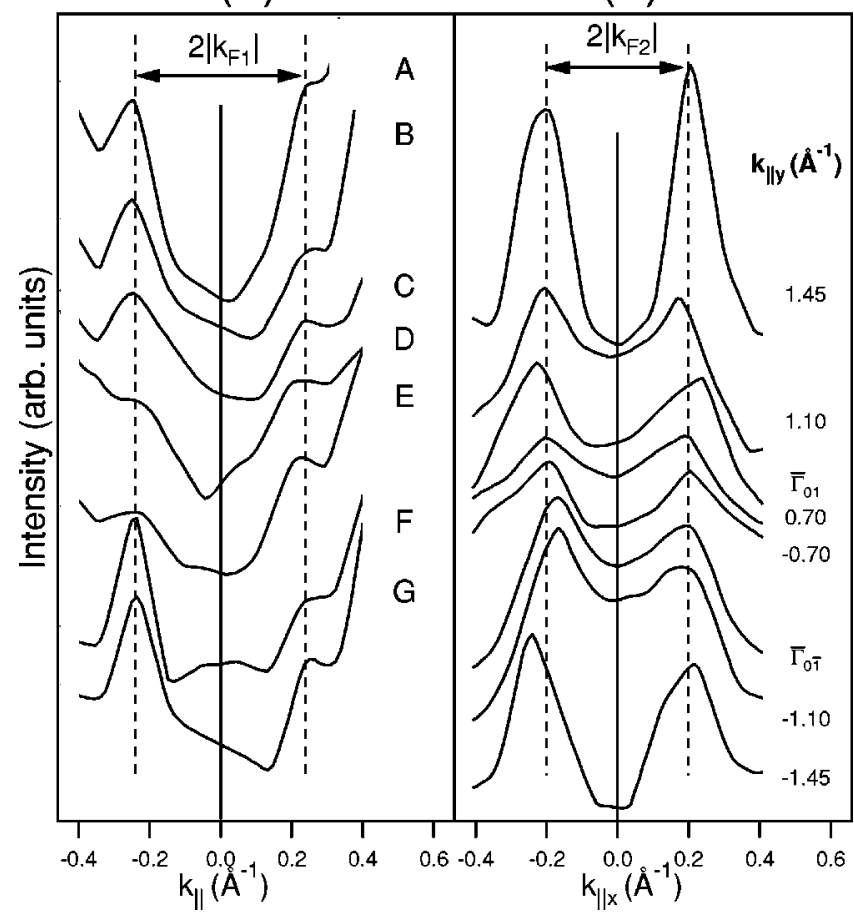

FIG. 7. Cuts of the FS mapping: (a) along the $2 \vec{k}_{F 1}$ direction. Labels correspond the regions where the cuts were performed [see Fig. 4(b)]. (b) Cuts along $2 \vec{k}_{F 2}$ direction, perpendicular to the $\overline{\Gamma Y}$ direction. Numbers indicate the $k_{\| y}$ values where the cuts were done.

Considering the hidden-nesting model, the $q_{C D W}$ vector $2 \vec{k}_{F 1}$ of the first CDW transition links states located in the crosslike sheets of the FS [see Fig. 4(b)]. In order to extract the value of $2\left|\vec{k}_{F 1}\right|$, different FS cuts along the $\vec{k}_{F 1}$ direction in different zones of the reciprocal space were made.

In Fig. 4(b) several arrows (from $A$ to $G$ ) have been indicated in order to identify the regions where the FS cuts have been done. It should be noted that each arrow represents the direction were several parallel cuts have been taken through. In this way, the influence of the modulation also present in these crosslike features was averaged. Some relevant cuts are plotted in Fig. 7(a), where the labels correspond to the labels in Fig. 4(b). The extracted mean values of $2\left|\vec{k}_{F 1}\right|$ for each of the zones are shown in Table II. From these results, we obtain an experimental mean value of $2\left|\vec{k}_{F 1}\right|=0.48 \pm 0.07 \AA^{-1}$. This is in agreement with the result $0.482 \pm 0.005 \AA^{-1}$ measured with x-ray diffraction by Ludecke et al.

TABLE II. Experimental distances obtained from FS map cuts $\left(\left|k_{F 1}\right|\right)$ between the maxima along the $\vec{k}_{F 1}$ direction. The letters that accompany the different cuts correspond to the labels in Fig. 4. The values are expressed in $\AA^{-1}$. The experimental error is $\pm 0.07 \AA^{-1}$.

\begin{tabular}{cccccccc}
\hline \hline Cut & $\left|k_{F 1}\right|$ & Cut & $\left|k_{F 1}\right|$ & Cut & $\left|k_{F 1}\right|$ & Cut & $\left|k_{F 1}\right|$ \\
\hline $\mathrm{A}$ & 0.49 & $\mathrm{~B}$ & 0.50 & $\mathrm{C}$ & 0.49 & $\mathrm{D}$ & 0.47 \\
$\mathrm{E}$ & 0.47 & $\mathrm{~F}$ & 0.47 & $\mathrm{G}$ & 0.50 & & \\
\hline \hline
\end{tabular}

Regarding the comparison of the measured nesting vectors values with the theoretical calculations, our experimental data agree much better with the $a b$ initio predictions than with the tight-binding results. In the case of $2 \vec{k}_{F 2}$, it is possible to infer it directly from Fig. 5(a). Here we can clearly observe how the vertical bright lines are better reproduced by the solid lines than by the dashed ones. This is even more evident by comparing the experimentally obtained $2\left|\vec{k}_{F 2}\right|$ $=0.40 \AA^{-1}$ with the corresponding values obtained by $a b$ initio and tight-binding results, $0.40 \AA^{-1}$ and $0.52 \AA^{-1}$, respectively. The nesting vector $2 \vec{k}_{F 1}$ is found to follow a similar behavior: the vector that links the crosslike sheets is better reproduced by the former theory. This better accord with first-principle calculations than with the extended Hückel ones has recently been observed in the case of the $\mathrm{K}_{0.3} \mathrm{MoO}_{3}$ blue bronze as well. ${ }^{30}$

We conclude that the values of the nesting vectors agree well with the expected ones in order to stabilize a CDW phase transition.

\section{CONCLUSION}

The normal state FS of the $m=4$ member of MPTB has been studied by ARPES. Two kinds of features are detected in the topology of the FS: a pair of parallel lines along the $\overline{\Gamma Y}$ direction and a crosslike feature centered around $\bar{M}$ points. Both shapes exhibit large flat areas which are very suitable for nesting. These results are compared with existing tight-binding ${ }^{10}$ and $a b$ initio $^{23}$ theoretical calculations. They are in agreement with the predictions of superimposed sheets that, according to the hidden nesting model, contribute to the FS. The FS splitting predicted as a consequence of interlayer interaction in $a b$ initio calculations ${ }^{23}$ is also observed in our experimental FS maps. In addition, we have also determined the modulation observed in the straight features appearing in the FS.

The nesting vectors of both phase transitions, $2\left|\vec{k}_{F 1}\right|$ and $2\left|\vec{k}_{F 2}\right|$, have been obtained from the FS mapping measured by ARPES. The values found are in excellent agreement with existing x-ray diffraction measurements. ${ }^{17}$ The nesting vectors predicted by ab initio calculations, in better accord with our experimental results, are smaller than those derived from tight binding.

We conclude that the favorable nesting conditions and the values of the Fermi vectors support that this is the mechanism responsible for the CDW instability, and corroborate the hidden-nesting model.

\section{ACKNOWLEDGMENTS}

We thank O. Pérez and Ph. Labbé for providing us with monophosphate tungsten bronzes single crystals. This work was financed by the Spanish "Ministerio de Ciencia y Tecnología" under Grant No. MAT2002-03431. A.M. acknowledges "Ministerio de Educacion y Cultura" and L.R. acknowledges "Ministerio de Ciencia y Tecnología" (Grants Nos. PF 0051683701 and ES2001-0106, respectively) for financial support. 
*Present address: Dpto. Física de Materiales, Universidad Complutense de Madrid, Av. Complutense s/n, 28040 Madrid, Spain.

${ }^{1}$ M. Greenblatt et al., Int. J. Mod. Phys. B 7, 3937 (1993).

${ }^{2}$ Physics and Chemistry of Low Dimensional Inorganic Conductors, in Vol. 354 of NATO Advanced Studies Institute Series B: Physics, edited by C. Schlenker, J. Dumas, M. Greenblatt, and S. Van Smaalen (Plenum, New York, 1996).

${ }^{3}$ S. Kagoshima, H. Nagasawa, and T. Sambongi, One-Dimensional Conductors (Springer-Verlag, Berlin, 1998).

${ }^{4}$ D. Jerome and H.J. Schultz, Adv. Phys. 32, 299 (1982).

${ }^{5}$ J. P. Pouget, in Highly Conducting Quasi One-Dimensional Organic Crystals, Semiconductors and Semi-metals, edited by E. M. Conwell (Pergamon, New York, 1988), Vol. 354.

${ }^{6}$ J.A. Wilson, F.J. Di Salvo, and S. Mahajan, Adv. Phys. 24, 117 (1975).

${ }^{7}$ A. Meerchaut and J. Rouxel, in Crystal Chemistry and Properties of Materials with Quasi One-Dimensional Structures, edited by J. Rouxel (Reidel, Dordrecht, 1996).

${ }^{8}$ M.-H. Whangbo, E. Canadell, P. Foury, and J.-P. Pouget, Science 252, 96 (1991).

${ }^{9}$ E. Wang, M. Greenblatt, I.E.-I. Rachidi, E. Canadell, M.-H. Whangbo, and S. Vadlamannati, Phys. Rev. B 39, 12969 (1989).

${ }^{10}$ E. Canadell and M.-H. Whangbo, Phys. Rev. B 43, 1894 (1991).

${ }^{11}$ E. Canadell, M.-H. Whangbo, C. Schlenker, and C. EscribeFilippini, Inorg. Chem. 28, 1466 (1989).

${ }^{12}$ E. Canadell, M.-H. Whangbo, and I.E.-I. Rachidi, Inorg. Chem. 29, 3871 (1990).

${ }^{13}$ G.-H. Gweon, J.W. Allen, J.A. Clack, Y.-X. Zhang, D.M. Poirier, P.J. Benning, C.G. Olson, J. Marcus, and C. Schlenker, Phys. Rev. B 55, R13 353 (1997).

${ }^{14}$ C. Hess, C. Schlenker, and J. Dumas, Phys. Rev. B 54, 4581 (1996).
${ }^{15}$ C. Schlenker, C. Hess, C. Le Touze, and J. Dumas, J. Phys. I 6, 2061 (1996).

${ }^{16}$ A. Ottolenghi and J.P. Pouget, J. Phys. I 6, 1059 (1996).

${ }^{17}$ J. Ludecke, A. Jobst, and S. van Smaalen, Europhys. Lett. 49, 357 (2000).

${ }^{18}$ A. Mascaraque, L. Roca, J. Avila, S. Drouard, H. Guyot, and M.C. Asensio, Phys. Rev. B 66, 115104 (2002).

${ }^{19}$ Z.S. Teweldemedhin, K.V. Ramanujachary, and M. Greenblatt, Phys. Rev. B 46, 7897 (1992).

${ }^{20}$ K.E. Smith, Annu. Rep. Prog. Chem., Sect. C: Phys. Chem. 90, 115 (1995).

${ }^{21}$ P. Roussel, O. Pérez, and Ph. Labbé, Acta Crystallogr., Sect. B: Struct. Sci. 57, 603 (2001).

${ }^{22}$ J.B. Goodenough, Bull. Soc. Chim. Fr. 1965, 1200; Czech. J. Phys., Sect. B 17, 304 (1967).

${ }^{23}$ E. Sandre, P. Foury, S. Ravy, and J.-P. Pouget, Phys. Rev. Lett. 86, 5100 (2001).

${ }^{24}$ A. Santoni, L.J. Terminello, F.J. Himpsel, and T. Takahashi, Appl. Phys. A: Solids Surf. 52, 299 (1991).

${ }^{25}$ N. Witkowski, M. Garnier, D. Purdie, Y. Baer, D. Malterre, and D. Groult, Solid State Commun. 103, 471 (1997).

${ }^{26}$ E.L. Shirley, L.J. Terminello, A. Santoni, and F.J. Himpsel, Phys. Rev. B 51, 13614 (1995).

${ }^{27}$ M.C. Asensio, J. Avila, L. Roca, A. Tejeda, G.D. Gu, M. Lindroos, B. Markiewicz, and A. Bansil, Phys. Rev. B 67, 014519 (2003).

${ }^{28}$ M. Lindroos, S. Sahrakorpi, and A. Bansil, Phys. Rev. B 65, 054514 (2002)

${ }^{29}$ G. Grüner, Density Waves in Solids (Addison-Wesley, Reading, 1994).

${ }^{30}$ J.L. Mozos, P. Ordejón, and E. Canadell, Phys. Rev. B 65, 233105 (2002). 